\title{
Neutrino Masses and Leptogenesis
}

\author{
by Ernest Ma \\ Physics Department, University of California, Riverside, CA 92521, USA \\ E-mail: ma@phyun8.ucr.edu
}

ABstract: I discuss the connection between neutrino masses and leptogenesis. I use three prime examples: (1) canonical seesaw, (2) triplet Higgs, and (3) $R$ parity violation.

\section{KEYwORDS: ineutrino mass, leptogenesis, $R$ parity'.}

\section{Origin of Neutrino Mass}

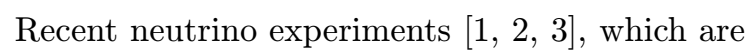
most naturally explained by neutrino oscillations, are strong indications that neutrinos are massive and mix with one another. So where do neutrino masses come from? In the minimal Standard Model, neutrinos appear only as left-handed fields in three electroweak doublets $\left(\nu_{i}, l_{i}\right)_{L}$, where $i=1,2,3$ is the family index. Charged leptons $l_{i}$ have right-handed components which are singlets, but not neutrinos. Hence neutrinos are massless two-component fermions, as long as there is no physics beyond the minimal Standard Model. Otherwise, there may be an effective dimension- 5 operator [4] $\left[\begin{array}{l}4 \\ 1\end{array}\right]$

$$
\frac{1}{\Lambda}\left(\nu_{i} \phi^{0}-l_{i} \phi^{+}\right)\left(\nu_{j} \phi^{0}-l_{j} \phi^{+}\right),
$$

where $\left(\phi^{+}, \phi^{0}\right)$ is the standard Higgs doublet and $\Lambda$ is a large mass, which yields a nonvanishing Majorana neutrino mass matrix as $\phi^{0}$ acquires a nonzero vacuum expectation value in the spontaneous breaking of the $S U(2) \times U(1)$ electroweak gauge symmetry.

All models of neutrino mass with the same low-energy particle content as that of the minimal Standard Model differ only in the way the above effective operator is realized [iñ $]$. The most well-known such model by far is the canonical seesaw model [i[6] $]$, where three right-handed neutrino singlets with large Majorana masses are added. This amounts to inserting a heavy internal fermion line between the two singlet factors of Eq. (1.1). The corresponding diagram can be read off to obtain the neutrino mass matrix as

$$
\left(\mathcal{M}_{\nu}\right)_{i j}=\frac{f_{i} f_{j}\left\langle\phi^{0}\right\rangle^{2}}{m_{N}}
$$

where $f_{i}$ are the Yukawa couplings linking $\nu_{i}$ with the heavy singlet $N$ with mass $m_{N}$. Obviously, we need three $N$ 's to obtain three naturally small seesaw masses for $\nu_{e}, \nu_{\mu}$, and $\nu_{\tau}$. On the other hand, other mechanisms are available [i5-1], the simplest alternative being the addition of a heavy scalar triplet $[\bar{i} \mathbf{1}]$. This is easily recognized if we rewrite Eq. (1.1) as

$$
\frac{1}{\Lambda}\left[\nu_{i} \nu_{j} \phi^{0} \phi^{0}-\left(\nu_{i} l_{j}+l_{i} \nu_{j}\right) \phi^{0} \phi^{+}+l_{i} l_{j} \phi^{+} \phi^{+}\right] \text {, }
$$

hence an insertion of the heavy scalar triplet

$$
\xi=\left(\xi^{++}, \xi^{+}, \xi^{0}\right)
$$

into the above yields a neutrino mass matrix given by

$$
\left(\mathcal{M}_{\nu}\right)_{i j}=\frac{2 f_{i j} \mu\left\langle\phi^{0}\right\rangle^{2}}{m_{\xi}^{2}},
$$

where $f_{i j}$ are the Yukawa couplings of $\xi$ to the lepton doublets and $\mu$ is its coupling (with the dimension of mass) to the scalar doublets. Note that Eq. (1.5) can also be interpreted as due to $\left\langle\xi^{0}\right\rangle$, i.e. [īi]

$$
\left\langle\xi^{0}\right\rangle=\frac{\mu\left\langle\phi^{0}\right\rangle^{2}}{m_{\xi}^{2}}<<m_{\xi} .
$$

This shows explicitly that the vacuum expectation value of a heavy scalar field may in fact be very small. 


\section{Leptogenesis}

Given that lepton number is not conserved in models of Majorana neutrino masses, the attractive possibility exists that a primordial lepton asymmetry may be created in the early Universe, which then gets converted into the present observed baryon asymmetry through the $B+L$ violating, but $B-L$ conserving interactions of the electroweak sphalerons [i]1]. In the canonical seesaw model, this is accomplished [ig] by the decays of $N_{i}$. Being Majorana fermions, $N_{1,2}$ may decay into either $l^{-} \phi^{+}$with $L=1$ or $l^{+} \phi^{-}$ with $L=-1$. Consider now the amplitude for $N_{1} \rightarrow l^{+} \phi^{-}$. It is the sum of 3 terms: the obvious tree graph, the one-loop vertex correction with

$$
N_{1} \rightarrow l^{-} \phi^{+} \rightarrow \phi^{-} l^{+}
$$

through the exchange of $N_{2}$, and the one-loop self-energy correction with

$$
N_{1} \rightarrow l^{-} \phi^{+} \rightarrow N_{2} \rightarrow l^{+} \phi^{-} .
$$

Let this amplitude be denoted by $A+i B$, where $B$ is the absorptive part, i.e. from putting the intermediate state $l^{-} \phi^{+}$on the mass shell. Then the lepton asymmetry from the decay of $N_{1}$ is proportional to

$$
|A+i B|^{2}-\left|A^{*}+i B^{*}\right|^{2}=4 \operatorname{Im}\left(A^{*} B\right) .
$$

This means that $C P$ violation is essential and that the presence of a different $N$, i.e. $N_{2}$, in the loop is necessary for leptogenesis.

Since 1995, there has been a resurgence of activity [10 107 in this topic. Consider $N_{1,2}$ and the mass matrix linking $\left(\bar{N}_{1 L}, \bar{N}_{2 L}, \bar{N}_{1 R} \bar{N}_{2 R}\right)$ with $\left(N_{1 L}, N_{2 L}, N_{1 R}, N_{2 R}\right)$ :

$$
\mathcal{M}_{N}=\left[\begin{array}{cc}
0 & \mathcal{M} \\
\tilde{\mathcal{M}} & 0
\end{array}\right]
$$

where

$$
\begin{aligned}
& \mathcal{M}=\left[\begin{array}{cc}
M_{1}+H_{11} & H_{12} \\
H_{12} & M_{2}+H_{22}
\end{array}\right], \\
& \tilde{\mathcal{M}}=\left[\begin{array}{cc}
M_{1}+H_{11} & \tilde{H}_{12} \\
\tilde{H}_{12} & M_{2}+H_{22}
\end{array}\right],
\end{aligned}
$$

and

$$
H_{12}=\left[M_{1} \sum_{\alpha} h_{\alpha 1}^{*} h_{\alpha 2}+M_{2} \sum_{\alpha} h_{\alpha 1} h_{\alpha 2}^{*}\right]
$$

$$
\begin{aligned}
& \times\left[g_{\alpha 12}^{d i s p}-\frac{i}{32 \pi}\right] \\
\tilde{H}_{12}= & {\left[M_{1} \sum_{\alpha} h_{\alpha 1} h_{\alpha 2}^{*}+M_{2} \sum_{\alpha} h_{\alpha 1}^{*} h_{\alpha 2}\right] } \\
& \times\left[g_{\alpha 12}^{d i s p}-\frac{i}{32 \pi}\right] \\
H_{j j}= & 2 M_{j} \sum_{\alpha}\left|h_{\alpha j}\right|^{2}\left[g_{\alpha j j}^{d i s p}-\frac{i}{32 \pi}\right]
\end{aligned}
$$

Note that $\tilde{H}_{12} \neq H_{12}^{*}$ because of the absorptive contribution, hence $\mathcal{M}_{N}$ is not hermitian. This is analogous to $\bar{K}-K$ or $\bar{B}-B$ mixing when the decay of the particles is also taken into account. The self-energy correction to $\mathcal{M}_{N}$ implies that mass eigenstates need not be $C P$ eigenstates, i.e. indirect $C P$ violation as exemplified by the $\epsilon$ parameter. The vertex correction to $\mathcal{M}_{N}$ implies that $C P$ may be violated in the decay itself, i.e. direct $C P$ violation as exemplified by the $\epsilon^{\prime}$ parameter. $N_{1}-N_{2}$ oscillations may also occur. However, all these things happen in an expanding Universe, i.e in a dense, hot medium which is changing with time, hence the exact details are complicated and are still being worked out [1 10 ind.

The primordial lepton asymmetry is generated from the decay of the lightest $N$, i.e. $N_{1}$ :

$$
\delta \simeq \frac{G_{F}}{2 \pi \sqrt{2}} \frac{1}{\left(m_{D}^{\dagger} m_{D}\right)_{11}} \sum_{j=2,3} \operatorname{Im}\left(m_{D}^{\dagger} m_{D}\right)_{1 j}^{2} \frac{M_{1}}{M_{j}},
$$

where $m_{D}$ is the Dirac mass matrix linking $\nu$ with $N$, and $M_{1}<<M_{j}$ has been assumed. This expression [1] 1, can then be used to study neutrino masses and mixing from atmospheric [1]1] and solar [i] neutrino oscillations and to extract information [13] on $N_{1}$. The range $10^{9}-$ $10^{13} \mathrm{GeV}$ for $M_{1}$ is found to be consistent with $n_{B} / g_{*} n_{\gamma} \sim 10^{-10}$. Since the self-energy contribution is proportional to $M_{1} /\left(M_{2}-M_{1}\right), \delta$ may be enhanced if $M_{2}-M_{1}<<M_{1}$, but the limit

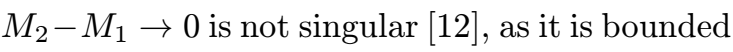
by the decay width of $N_{1}$.

\section{Triplet Higgs Model}

If neutrino masses come from heavy triplet scalars $\left.[\overline{7}]_{1}\right]$, then the mixing of $\xi_{1}$ and $\xi_{2}$ through their absorptive parts, i.e. self-energy contributions, leads to the physical mass eigenstates $\psi_{1}$ and $\psi_{2}$ 
which are not $C P$ eigenstates. Their decay asymmetries are given by

$$
\delta_{i} \simeq \frac{\operatorname{Im}\left[\mu_{1} \mu_{2}^{*} \sum_{k, l} f_{1 k l} f_{2 k l}^{*}\right]}{8 \pi^{2}\left(M_{1}^{2}-M_{2}^{2}\right)} \frac{M_{i}}{\Gamma_{i}},
$$

and for $M_{1,2} \sim 10^{13} \mathrm{GeV}$, realistic neutrino masses and leptogenesis are possible.

\section{Neutrino Masses from $R$ Parity Violation}

I now come to my third example which is the generation of neutrino masses through $R$ parity violation in supersymmetry [1] 19i]. The well-known superfield content of the Minimal Supersymmetric Standard Model (MSSM) is given by

$$
\begin{aligned}
Q_{i} & =\left(u_{i}, d_{i}\right)_{L} \sim(3,2,1 / 6), \\
u_{i}^{c} & \sim\left(3^{*}, 1,-2 / 3\right), \\
d_{i}^{c} & \sim\left(3^{*}, 1,1 / 3\right), \\
L_{i} & =\left(\nu_{i}, l_{i}\right)_{L} \sim(1,2,-1 / 2), \\
l_{i}^{c} & \sim(1,1,1) ; \\
H_{1} & =\left(\bar{\phi}_{1}^{0},-\phi_{1}^{-}\right) \sim(1,2,-1 / 2), \\
H_{2} & =\left(\phi_{2}^{+}, \phi_{2}^{0}\right) \sim(1,2,1 / 2) .
\end{aligned}
$$

Given the above transformations under the standard $S U(3) \times S U(2) \times U(1)$ gauge group, the corresponding superpotential should contain in general all gauge-invariant bilinear and trilinear combinations of the superfields. However, to forbid the nonconservation of both baryon number $(B)$ and lepton number $(L)$, each particle is usually assigned a dicrete $R$ parity:

$$
R \equiv(-1)^{3 B+L+2 j},
$$

which is assumed to be conserved by the allowed interactions. Hence the MSSM superpotential has only the terms $H_{1} H_{2}, H_{1} L_{i} l_{j}^{c}, H_{1} Q_{i} d_{j}^{c}$, and $\mathrm{H}_{2} Q_{i} u_{j}^{c}$. Since the superfield $\nu_{i}^{c} \sim(1,1,0)$ is absent, $m_{\nu}=0$ in the MSSM as in the minimal

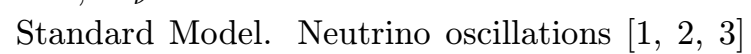
are thus unexplained.

Phenomenologically, it makes sense to require only $B$ conservation (to make sure that the proton is stable), but to allow $L$ violation (hence $R$ parity violation) so that the additional terms $L_{i} H_{2}, L_{i} L_{j} l_{k}^{c}$, and $L_{i} Q_{j} d_{k}^{c}$ may occur. Note that they all have $\Delta L=1$. Neutrino masses are now possible [1] 15 . From the bilinear terms

$$
-\mu H_{1} H_{2}+\epsilon_{i} L_{i} H_{2},
$$

a $7 \times 7$ neutralino-neutrino mass matrix is obtained:

$$
\left[\begin{array}{ccccc}
M_{1} & 0 & -g_{1} v_{1} & g_{1} v_{2} & -g_{1} u_{i} \\
0 & M_{2} & g_{2} v_{1} & -g_{2} v_{2} & g_{2} u_{i} \\
-g_{1} v_{1} & g_{2} v_{1} & 0 & -\mu & 0 \\
g_{1} v_{2} & -g_{2} v_{2} & -\mu & 0 & \epsilon_{i} \\
-g_{1} u_{i} & g_{2} u_{i} & 0 & \epsilon_{i} & 0
\end{array}\right]
$$

where $v_{1,2}=\left\langle\phi_{1,2}^{0}\right\rangle / 2$ and $u_{i}=\left\langle\tilde{\nu}_{i}\right\rangle / 2$, with $i=e, \mu, \tau$. Note first the important fact that a nonzero $\epsilon_{i}$ implies a nonzero $u_{i}$. Note also that even if $u_{i} / \epsilon_{i}$ is not the same for all $i$, only one linear combination of the three neutrinos gets a tree-level mass [1 $16 \overline{6}$ ]. From the trilinear terms, neutrino masses are also obtained, but now as one-loop radiative corrections. Note that these occur as the result of supersymmetry breaking and are suppressed by $m_{d}^{2}$ or $m_{l}^{2}$.

\section{5. $L$ Violation and the Universe}

As noted earlier, the $R$ parity violating interactions have $\Delta L=1$. Furthermore, the particles involved have masses at most equal to the supersymmetry breaking scale, i.e. a few $\mathrm{TeV}$. This means that their $L$ violation together with the $B+L$ violation by sphalerons [i any primordial $B$ or $L$ asymmetry of the Universe [i] $\overline{7}_{i}$. To avoid such a possibility, one may reduce the relevant Yukawa couplings to less than about $10^{-7}$, but a typical minimum value of $10^{-4}$ is required for realistic neutrino masses. Hence the existence of the present baryon asymmetry of the Universe is unexplained if neutrino masses originate from these $\Delta L=1$ interactions. This is a generic problem of all models of radiative neutrino masses where the $L$ violation can be traced to interactions occuring at energies below $10^{13}$ $\mathrm{GeV}$ or so.

\section{Leptogenesis from $R$ Parity Vio- lation}

Once the notion of $R$ parity violation is intro- 
duced, there are many new terms to be added in the Lagrangian. Some may be responsible for realistic neutrino masses and may even participate in the erasure of any primordial $B$ or $L$ asymmetry of the Universe, but others may be able to produce a lepton asymmetry on their own which then gets converted into the present observed baryon asymmetry of the Universe through the sphalerons. A recent proposal [1] $\left.{ }_{1}^{1}\right]$ shows how this may happen in a specific model.

Consider the usual $4 \times 4$ neutralino mass matrix in the $\left(\tilde{B}, \tilde{W}_{3}, \tilde{h}_{1}^{0}, \tilde{h}_{2}^{0}\right)$ basis:

$$
\left[\begin{array}{cccc}
M_{1} & 0 & -s m_{3} & s m_{4} \\
0 & M_{2} & c m_{3} & -c m_{4} \\
-s m_{3} & c m_{3} & 0 & -\mu \\
s m_{4} & -c m_{4} & -\mu & 0
\end{array}\right]
$$

where $s=\sin \theta_{W}, c=\cos \theta_{W}, m_{3}=M_{Z} \cos \beta$, $m_{4}=M_{Z} \sin \beta$, and $\tan \beta=v_{2} / v_{1}$. The above assumes that $\epsilon_{i}$ and $u_{i}$ are negligible in Eq. (4.10), which is a good approximation because neutrino masses are so small. We now choose the special case of

$$
m_{3}, m_{4}<<M_{2}<M_{1}<\mu \text {. }
$$

As a result, the two higgsinos $\tilde{h}_{1,2}^{0}$ form a heavy Dirac particle of mass $\mu$ and the other two less heavy Majorana fermion mass eigenstates are

$$
\begin{aligned}
\tilde{B}^{\prime} & \simeq \tilde{B}+\frac{s c \delta r_{1}}{M_{1}-M_{2}} \tilde{W}_{3}+\ldots \\
\tilde{W}_{3}^{\prime} & \simeq \tilde{W}_{3}-\frac{s c \delta r_{2}}{M_{1}-M_{2}} \tilde{B}+\ldots
\end{aligned}
$$

where $\delta=M_{Z}^{2} \sin 2 \beta / \mu$, and

$$
r_{1,2}=\frac{1+M_{1,2} / \mu \sin 2 \beta}{1-M_{1,2}^{2} / \mu^{2}} .
$$

We now observe that whereas $\tilde{B}$ couples to both $\bar{l}_{L} \tilde{l}_{L}$ and $\bar{l}_{L}^{c} \tilde{l}_{L}^{c}, \tilde{W}_{3}$ couples only to $\bar{l}_{L} \tilde{l}_{L}$ because $l_{L}^{c}$ is trivial under $S U(2)_{L}$. On the other hand, $R$ parity violation implies that there is $\tilde{l}_{L}-h^{-}$mixing as well as $\tilde{l}_{L}^{c}-h^{+}$mixing. Therefore, both $\tilde{B}^{\prime}$ and $\tilde{W}_{3}^{\prime}$ decay into $l^{ \pm} h^{\mp}$ and may be the seeds of a lepton asymmetry in such a scenario.

Let the $\tilde{l}_{L}-h^{-}$mixing be very small (which is a consistent assumption for realistic neutrino masses from bilinear $R$ parity violation). Then
$\tilde{W}_{3}^{\prime}$ decays only through its $\tilde{B}$ component. Hence the decay rate of the LSP (Lightest Supersymmetric Particle), i.e. $\tilde{W}_{3}^{\prime}$, is very much suppressed, first by $\delta$ and then by the $\tilde{l}_{L}^{c}-h^{+}$mixing which will be denoted by $\xi$. Our construction is aimed at satisfying the out-of-equilibrium condition:

$$
\Gamma\left(\tilde{W}_{3}^{\prime} \rightarrow l^{ \pm} h^{\mp}\right)<H=1.7 \sqrt{g_{*}}\left(T^{2} / M_{P l}\right)
$$

at the temperature $T \sim M_{2}$, where $H$ is the Hubble expansion rate of the Universe with $g_{*}$ the effective number of massless degrees of freedom and $M_{P l}$ the Planck mass. This implies

$$
\left(\frac{\xi|\delta| r_{2}}{M_{1}-M_{2}}\right)^{2} \frac{1}{M_{2}}<1.9 \times 10^{-14} \mathrm{GeV}^{-1}
$$

where we have used $g_{*}=10^{2}$ and $M_{P l}=10^{18}$ $\mathrm{GeV}$.

The lepton asymmetry generated from the decay of $\tilde{W}_{3}^{\prime}$ has both vertex and self-energy loop contributions from the insertion of $\tilde{B}^{\prime}$. However, the coupling of $\tilde{B}^{\prime}$ to $l^{ \pm} h^{\mp}$ is suppressed only by $\xi$ and not by $\delta$, thus a realistic asymmetry may be established if $\xi$ is not too small. Let $x \equiv M_{2}^{2} / M_{1}^{2}$, then the decay asymmetry of $\tilde{W}_{3}^{\prime}$ is given by

$$
\epsilon=\frac{\alpha \xi^{2}}{2 \cos ^{2} \theta_{W}} \frac{\operatorname{Im} \delta^{2}}{|\delta|^{2}} \frac{\sqrt{x} g(x)}{1-x},
$$

where

$$
g(x)=1+\frac{2(1-x)}{x}\left[\left(\frac{1+x}{x}\right) \ln (1+x)-1\right] .
$$

The phase of $\delta$ comes from the relative phase between $M_{1}$ and $M_{2}$.

To make sure that at $T \sim M_{2}$, the $L$ violating processes $l^{ \pm} h^{\mp} \leftrightarrow l^{\mp} h^{ \pm}$through $\tilde{B}^{\prime}$ exchange do not erase $\epsilon$, we require

$$
\left(\frac{2 e^{2} \xi^{2}}{\cos ^{2} \theta_{W}}\right)^{2} \frac{1}{M_{1}^{2}} \frac{T^{3}}{32 \pi} \frac{f(x)}{(1-x)^{2}}<H
$$

at $T \sim M_{2}$, where

$$
\begin{gathered}
f(x)=1+\frac{2(1-x)}{x^{2}}[(1+3 x) \ln (1+x) \\
-x(1+x)],
\end{gathered}
$$

which implies

$$
\frac{\xi^{4}}{M_{2}} \frac{x f(x)}{(1-x)^{2}}<2.6 \times 10^{-14} \mathrm{GeV}^{-1} .
$$


A sample solution is

$$
\begin{aligned}
& M_{1}=3 \mathrm{TeV}, \frac{\delta}{M_{1}-M_{2}}=8.3 \times 10^{-4} \\
& M_{2}=2 \mathrm{TeV}, \xi=2 \times 10^{-3} .
\end{aligned}
$$

In that case,

$$
\epsilon=3.6 \times 10^{-8} \operatorname{Im} \delta^{2} /|\delta|^{2},
$$

and

$$
\frac{n_{B}}{g_{*} n_{\gamma}} \sim \frac{\epsilon}{3 g_{*}} \sim 10^{-10} \frac{\operatorname{Im} \delta^{2}}{|\delta|^{2}}
$$

Hence realistic leptogenesis is possible if $\xi \sim 10^{-3}$ can be obtained.

The origin of $\tilde{l}_{L}^{c}-h^{+}$mixing in $R$ parity violation is usually the term $H_{1} \tilde{L} \tilde{l}^{c}$, which is very small because $\langle\tilde{\nu}\rangle$ has to be very small. To obtain $\xi \sim 10^{-3}$, we need to add the nonholomorphic [1] $\left.{ }^{i}\right]$ term $H_{2}^{\dagger} H_{1} \tilde{l}^{c}$ which is generally unconstrained.

\section{Conclusion}

Given a mechanism for generating small Majorana neutrino masses, it is often a bonus to find that leptogenesis is possible at the same time. In the canonical seesaw and triplet Higgs models, the same new physics is responsible for both. In $R$ parity nonconserving supersymmetry, they may come from different sectors of the theory.

\section{Acknowledgments}

I thank Enrico Nardi and William Ponce and the other organizers for their great hospitality at Cartagena. This work was supported in part by the U. S. Department of Energy under Grant No. DE-FG03-94ER40837.

\section{References}

[1] Y. Fukuda et al., Phys. Lett. B433, 9 (1998); B436, 33 (1998); Phys. Rev. Lett. 81, 1562 (1998); 82, 2644 (1999).
[2] R. Davis, Prog. Part. Nucl. Phys. 32, 13 (1994); Y. Fukuda et al., Phys. Rev. Lett. 77, 1683 (1996); 81, 1158 (1998); 82, 1810, 2430 (1999); P. Anselmann et al., Phys. Lett. B357, 237 (1995); Erratum: B361, 235 (1996); J. N. Abdurashitov et al., Phys. Lett. B328, 234 (1994); Phys. Rev. Lett. 83, 4686 (1999).

[3] C. Athanassopoulos et al., Phys. Rev. Lett. 75, 2650 (1995); 77, 3082 (1996); 81, 1774 (1998).

[4] S. Weinberg, Phys. Rev. Lett. 43, 1566 (1979).

[5] E. Ma, Phys. Rev. Lett. 81, 1171 (1998).

[6] M. Gell-Mann, P. Ramond, and R. Slansky, in Supergravity, edited by P. van Nieuwenhuizen and D. Z. Freedman (North-Holland, Amsterdam, 1979), p. 315; T. Yanagida, in Proceedings of the Workshop on the Unified Theory and the Baryon Number in the Universe, edited by $\mathrm{O}$. Sawada and A. Sugamoto (KEK Report No. 7918, Tsukuba, Japan, 1979), p. 95; R. N. Mohapatra and G. Senjanovic, Phys. Rev. Lett. 44, 1316 (1980).

[7] E. Ma and U. Sarkar, Phys. Rev. Lett. 80, 5716 (1998).

[8] V. A. Kuzmin, V. A. Rubakov, and M. E. Shaposhnikov, Phys. Lett. 155B, 36 (1985).

[9] M. Fukugita and T. Yanagida, Phys. Lett. 174B, 45 (1986).

[10] M. Flanz, E. A. Paschos, and U. Sarkar, Phys. Lett. B345, 248 (1995); Erratum: B382, 447 (1996); M. Flanz, E. A. Paschos, U. Sarkar, and J. Weiss, Phys. Lett. B389, 693 (1996); W. Buchmuller and M. Plumacher, Phys. Lett. B389, 73 (1996); B431, 354 (1998); L. Covi, E. Roulet, and F. Vissani, Phys. Lett. B384, 169 (1996); L. Covi and E. Roulet, Phys. Lett. B399, 113 (1997); E. Roulet, L. Covi, and F. Vissani, Phys. Lett. B424, 101 (1998); A. Pilaftsis, Phys. Rev. D56, 5431 (1997); Int. J. Mod. Phys. A14, 1811 (1999); M. Flanz and E. A. Paschos, Phys. Rev. D58, 113009 (1998); R. Rangarajan and H. Mishra, Phys. Rev. D61, 043509 (2000); R. Barbieri, P. Creminelli, A. Strumia, and N. Tetradis, Nucl. Phys. B575, 61 (2000); G. Mangano and G. Miele, hepph/9912471; O. Lalakulich, E. A. Paschos, and M. Flanz, hep-ph/0002021.

[11] The numerical coefficient of $\delta$ differs from one another in several early references. The one I use here was first_given by Buchmuller and Plumacher in Ref.[10]. 
[12] J.-M. Frere, F.-S. Ling, M. H. G. Tytgat, and V. Van Elewyck, Phys. Rev. D60, 016005 (1999).

[13] G. Lazarides, Q. Shafi, and N. D. Vlachos, Phys. Lett. B427, 53 (1998); W. Buchmuller and T. Yanagida, Phys. Lett. B445, 399 (1999); J. Ellis, S. Lola, and D. V. Nanopoulos, Phys. Lett. B452, 87 (1999); M. S. Berger and B. Brahmachari, Phys. Rev. D60, 073009 (1999); A. S. Joshipura and E. A. Paschos, hep-ph/9906498; H. Goldberg, Phys. Lett. B474, 389 (2000).

[14] L. Hall and M. Suzuki, Nucl. Phys. B231, 419 (1984).

[15] R. Hempfling, Nucl. Phys. B478, 3 (1996); F. M. Borzumati, Y. Grossman, E. Nardi, and Y. Nir, Phys. Lett. B384, 123 (1996); H.-P. Nilles and N. Polonsky, Nucl. Phys. B484, 33 (1997); E. Nardi, Phys. Rev. D55, 5772 (1997); M. A. Diaz, J. C. Romao, and J. W. F. Valle, Nucl. Phys. B524, 23 (1998); M. Drees, S. Pakvasa, X. Tata, and T. ter Veldhuis, Phys. Rev. D57, R5335 (1998); B. Mukhopadhyaya, S. Roy, and F. Vissani, Phys. Lett. B443, 191 (1998); E. J. Chun, S. K. Kang, and C. W. Kim, Nucl. Phys. B544, 89 (1999); R. Adhikari and G. Omanovic, Phys. Rev. D59, 073003 (1999); O. C. W. Kong, Mod. Phys. Lett. A14, 903 (1999); S. Rakshit, G. Bhattacharyya, and A. Raychaudhuri, Phys. Rev. D59, 091701 (1999); K. Choi, E. J. Chun, and K. Hwang, Phys. Rev. D60, 031301 (1999); S. Y. Choi, E. J. Chun, S. K. Kang, and J. S. Lee, Phys. Rev. D60, 075002 (1999); L. Clavelli and P. H. Frampton, hep-ph/9811326; D. E. Kaplan and A. E. Nelson, J. High Energy Phys. 0001, 033 (2000); A. S. Joshipura and S. Vempati, Phys. Rev. D60, 111303 (1999); Y. Grossman and H. E. Haber, hep-ph/9906310; G. Bhattacharyya, H. V. Klapdor-Kleingrothaus, and H. Pas, Phys. Lett. B463, 77 (1999); A. Abada and M. Losada, hep-ph/9908352; O. Haug, J. D. Vergados, A. Faessler, and S. Kovalenko, Nucl. Phys. B565, 38 (2000); J. C. Romao, M. A. Diaz, M. Hirsch, W. Porod, and J. W. F. Valle, Phys. Rev. D61, 071703 (2000); E. J. Chun and S. K. Kang, Phys. Rev. D61, 075012 (2000); F. Takayama and M. Yamaguchi, Phys. Lett. B476, 116 (2000).

[16] C.-H. Chang and T.-F. Feng, Eur. Phys. J. C12, 137 (2000).

[17] B. A. Campbell, S. Davidson, J. E. Ellis, and K. Olive, Phys. Lett. B256, 457 (1991); W. Fischler, G. F. Giudice, R. G. Leigh, and S. Paban, Phys. Lett. B258, 45 (1991); H. Dreiner and G.
G. Ross, Nucl. Phys. B410, 188 (1993); J. M. Cline, K. Kainulainen, and K. A. Olive, Phys. Rev. D49, 6394 (1994); E. Ma, M. Raidal, and U. Sarkar, Phys. Lett. B460, 359 (1999).

[18] T. Hambye, E. Ma, and U. Sarkar, Phys. Rev. D62, 015010 (2000).

[19] L. J. Hall and L. Randall, Phys. Rev. Lett. 65, 2939 (1990); F. Borzumati, G. R. Farrar, N. Polonsky, and S. Thomas, Nucl. Phys. B555, 53 (1999). 